J. Clin. Chem. Clin. Biochem.

Vol. 16, 1978, pp. 103-110

\title{
Drug Interference in Clinical Chemistry: Studies on Ascorbic Acid
}

By G. Siest (Chairman), W. Appel, G. B. Blijenberg, B. Capolaghi, M. M. Galteau, C. Heusghem, M. Hjelm, K. L. Lauer ${ }^{1}$ ), B. Le Perron, V. Loppinet, C. Love, R. J. Royer, C. Tognoni and P. Wilding

Expert group "Drug Interference in Clinical Chemistry", Bureau Communautaire de Reference (Dr. K. L. Lauer), Commission des Communautés Europeénnes

(Received March 25/August 10, 1977)

Summary: The expert group "Drug Interference in Clinical Chemistry" of the Bureau of Reference, Directorate General for Research, Science and Education of the Commission of the European Communities, consisting of one participant of each member of the European Communities, presents this first report on the final results of its activities.

Within the framework of a first stage basic program, the paper describes interferences of therapeutic and elevated doses of ascorbic acid on commonly used clinical chemical methods. This is the result of a bipartite study that was jointly planned, carried out and evaluated. Local and personal influences have been eliminated, as have variations due to methodology, measurement equipment and reagents, in order to be able to present distinct causal effects of ascorbic acid.

No definite influence of ascorbic acid on analytical values for urea, cholesterol, calcium, protein, bilirubin, aspartate aminotransferase and alkaline phosphatase could be detected.

At therapeutic concentrations, ascorbic acid distinctly interferes with the analysis of glucose, uric acid, creatinine and inorganic phosphate. The extent and direction of interferences vary, depending on the type of reaction, kit and apparatus. In some cases the influence of ascorbic acid results in severe disturbance of the analytical methods leading to useless values.

\section{Arzneimittelstörungen in der Klinischen Chemie: Untersuchungen mit Ascorbinsäure}

Zusammenfassung: Die Expertengruppe “Drug Interference in Clinical Chemistry" des "Büro für Standardisierung" im Generalsekretariat für Forschung, Wissenschaft und Erziehung der Europäischen Gemeinschaft, legt erste Abschlußergebnisse ihrer Tätigkeit vor. Im Rahmen eines vorab erarbeiteten Grundsatzprogrammes werden in einer gemeinsam geplanten, durchgeführten und ausgewerteten zweiteiligen Studie Störeinflüsse von therapeutischen und hohen Dosen von Ascorbinsäure auf gebräuchliche klinisch-chemische Untersuchungsmethoden beschrieben.

Nicht oder statistisch nicht sicherbar beeinflußt werden Bestimmungsmethoden für Harnstoff, Cholesterin, Calcium, Protein, Bilirubin, Aspartataminotransferase und alkàlische Phosphatase.

Eindeutige Beeinfluṣsungen bereits bei therapeutișchen Ascorbinșäurekombinationen liegen vor bei Glucose, Harnsäure, Kreatinin und anorganịschem Phosphat. Ausmaß und Richtung der Störung variieren in Abhängigkeit von Reaktionstyp, Reagenzienkombination und Gerät. In einigen Fällen führt der Einfluß der Ascorbinsäure zu schweren Störungen der analytischen Methode bis zur Unḅrauchbarkeit der Werte.

\section{Introduction}

The development of a wide range of biological tests, mechanisation, and data processing have contributed to the discovery of many factors that cause variations

1) Dr. Lauer died in August 1976. We are deeply grateful for his everlasting assistance. in laboratory tests. Drugs are predominant among these factors, owing to their interference with analytical methods, as well as their pharmacological effects; there is an extensive literature on this problem $(1-19)$.

Indeed, the interpretation of a laboratory result using colorimetric, spectrophotometric, fluorimetric or en- 
zymatic methods may be difficult and even erroneous due to the following factors:

\section{Analytical factors}

Quality control is designed to detect most of these errors. The evolution towards more accurate and more specific techniques will make it possible to avoid, or at least control such errors.

\section{Physiological or environmental factors}

Variations due to age, sex, exercise, meal intake etc. are now well known, but the interference of environmental factors, such as climatic conditions, dietary habits, pesticides, and, of course, drugs, is also considerable and often more important.

With respect to environmental factors, it was considered necessary to initiate a systematic study of analytical interferences.

For this purpose a working sub-group "Drug Interference in Clinical Chemistry" (20) was founded by the Bureau of Reference, (Dr. Lauer) Directorate General for Research, Science and Education of the Commission of the European Communities. This group of experts consists of delegates of the national clinical chemical societies.

Within the framework of this group of experts in the European Communities the following goals shall be pursued:

\section{Establish and keep an up-to-date list of drugs which} are likely to effect reference and routine methods.

2. Prepare a protocol for the study of analytical interferences.

3. Seek out the methods least sensitive to analytical interference from drugs and their metabolites.

4. Prepare a control serum containing drugs and metabolites.

5. Establish the percentage interference due to drugs, to be used by clinical chemists and clinicians for correct interpretation of laboratory results.

Details concerning general rules, guide lines and first results up to 1975 , have already been presented by Siest, Lauer et. al. (20) at Pont à Mousson and by Appel, Lauer et al. at Munich, 1976 (21).

In this paper results relevant to points 2-5 above are presented. Ascorbic acid was selected in preference to acetylsalicylic acid as the first substance to be investigated by the above mentioned expert group. It was necessary to test a simple drug molecule that was easy to find commercially in a pure state, and that was known to interfere with certain tests used in clinical chemistry. Ascorbic acid fulfills these criteria and was chosen as a model for the general protocol.

\section{Materials and Methods}

For this study, commercially available ascorbic acid (no special lot) and commercial, lyophilized human serum were chosen. An ascorbic acid overdose was simulated by mixing the control serum (redissolved in distilled water) and an "overloaded serum" (redissolved in an aqueous solution of ascorbic acid) (See Annexe "General protocol").

Data on the parameters studied, analytical methods; apparatus and technical equipment are given in table 1.

First study

The concentrations tested during the first study ranged from $0-2 \mathrm{~g} / 1$ of ascorbic acid. Methods listed in table 1 were studied. Measurements were performed in duplicate on each overloaded solution.

The sera were prepared daily, and the analyses were carried out on 5 consecutive days by routine methods within routine work at 8 laboratories of different members of the expert group in different countries of the European Communities. The results are presented in $\mu \mathrm{mol} / \mathrm{l}$ or in mmol/l depending upon the parameter, accompanied by specifications of the equipment used, techniques and variation coefficient in the zone of values measured.

Since the parameters showed a more or less clear interference, additional statistical studies were carried out (table).

- Test of significance between control (dose 0 ) and the first ascorbic acid overdose $(0.71 \mathrm{mmol} / \mathrm{l})$ for each laboratory, for all laboratories together, regrouping laboratories according to the techniques used.

- A 2-factor variance analysis was performed for each laboratory result. This made it possible to determine the significance of the difference between the control serum and the first serum (first concentration). Other analyses of variance were performed on the same parameters: one by regrouping all the laboratories, the other by regrouping laboratories using the same techniques (tab. 2).

Second study

This study was then extended in 4 laboratories, in order to identify the parameters that cause a distinct interference. Additional assays were performed at the following concentrations that are close to the therapeutic values: $0.14 \mathrm{mmol} / \mathrm{l}$, $0.34 \mathrm{mmol} / 1,0.71 \mathrm{mmol} / \mathrm{l}, 1.42 \mathrm{mmol} / \mathrm{l}$. On the basis of this study with ascorbic acid, we have established a general protocol for the study of drug interference (cf. annexe).

The distribution of protocols and questionaires, samples of ascorbic acid and serum to each participant of the study, the collection and mathematical-statistical evaluation of experimental data and the preparation of results were performed under the supervision of the Chairman of the group, Dr. Siest (14). Discussion of results took place at the group's meetings at Geneva and Pont à Mousson.

\section{Results}

The evaluated "General protocol of analytical interference by ascorbic acid" is shown in the annexe.

\section{First study}

For certain parameters, no comment is called for, either because there is no interference, i.e.

Urea, Cholesterol, Calcium and Protein;

or because the dispersion is too great and the variations are not systematic, i.e.

Bilirubin, Aspartate Aminotransferase; or because of the technical problems encountered, i.e.

Alkaline Phosphatase. 
Tab. 1. Methods: Objectives, analytical methods, apparatus, and technical equipment.

\begin{tabular}{|c|c|c|}
\hline Objectives & Method & Instrument \\
\hline Aspartate aminotransferase & $\begin{array}{l}\text { UV-test, kinetic, opt. } \\
\text { UV-test, kinet. non opt. } \\
\text { UV-test, end point } \\
\text { Colorimetric test }\end{array}$ & $\begin{array}{l}\text { Eppendorf } 5020 \\
\text { LKB } 8600 \\
\text { ABA } 100 \\
\text { Greiner GSA II } \\
\text { SMA } 12 / 60 \\
\text { Autochemist } \\
\text { ACA DuPont }\end{array}$ \\
\hline Phosphatase, alkaline & $\begin{array}{l}\text { Kinetic-test, } p \text {-nitrophenyl phosphate, buffer: diethanolamine } \\
\text { Kinetic-test, } p \text {-nitrophenyl phosphate, buffer: glycine } \\
\text { End-point-test, } p \text {-nitrophenyl phosphate, buffer: 2-amino- } \\
\text { 2-methyl-1-propanol } \\
\text {-End-point-test, } p \text {-nitrophenyl phosphate } \\
\text { End-point-test, phenyl disodium phosphate }\end{array}$ & $\begin{array}{l}\text { Eppendorf } 5020 \\
\text { LKB } 8600 \\
\text { SMA } 12 / 60 \\
\text { ACA DuPont } \\
\text { Greiner GSA II } \\
\text { Beckman DSA } \\
\text { SMA } 12 / 60 \\
\text { Autochemist }\end{array}$ \\
\hline Bilirubin & $\begin{array}{l}\text { Sulfanilic acid-caffein } \\
\text { Dichloraniline-nitrite }\end{array}$ & $\begin{array}{l}\text { SMAC } \\
\text { SMA } 12 / 60 \\
\text { Eppendorf } 1101 \\
\text { Beckman DSA } \\
\text { Autochemist } \\
\text { Bilirubinometer } \\
\text { Greiner GSA II }\end{array}$ \\
\hline Calcium & $\begin{array}{l}\text { o-Cresolphthalein-complexon } \\
\text { Flame photometry } \\
\text { Thymolphthalein-complexon } \\
\text { Calcein }\end{array}$ & $\begin{array}{l}\text { SMA } 12 / 60 \\
\text { ACA DuPont } \\
\text { SMAC } \\
\text { Zeiss PF } 5 \\
\text { Autochemist } \\
\text { Greiner GSA II } \\
\text { Oxford Titrator }\end{array}$ \\
\hline Cholesterol & $\begin{array}{l}\text { Acetic anhydride-acetic acid Liebermann-Burchard, Huang, } \\
\text { Watson } \\
\text { Esterase/oxidase-catalase } \\
\text { Esterase-hydrogen peroxidase }\end{array}$ & $\begin{array}{l}\text { SMA } 12 / 60 \\
\text { Eppendorf } 1101 \\
\text { Autochemist } \\
\text { LKB } 7400 \\
\text { ABA } 100 \\
\text { Greiner USA II }\end{array}$ \\
\hline Creatinine & $\begin{array}{l}\text { Alkaline picrate (Jaffé) without deproteinisation } \\
\text { Alkaline picrate (Jaffé) with dialysis } \\
\text { Picric acid, kinetic test }\end{array}$ & $\begin{array}{l}\text { Autochemist } \\
\text { SMA } 12 / 60 \\
\text { LKB } 8600 \\
\text { Eppendorf } 5020\end{array}$ \\
\hline Glucose & $\begin{array}{l}\text { Glucose oxidase-Perid } \\
\text { Glucose oxidase-peroxidase } \\
\text { Glucose oxidase-Trinder method } \\
\text { Neocuproin }\end{array}$ & $\begin{array}{l}\text { SMA } 12 / 60 \\
\text { Vitatron UC } 200 \mathrm{~S} \\
\text { LKB } 7400 \\
\text { Gilford } \\
\text { SMA } 12 / 60\end{array}$ \\
\hline Phosphate, inorganic & $\begin{array}{l}\text { Molybdate-hydroquinone-ascorbate } \\
\text { Molybdate-p-methylaminophenolsulfate } \\
\text { Molybdate-vanadate } \\
\text { Molybdic acia-stänous chloride-hydrazine }\end{array}$ & $\begin{array}{l}\text { Autochemist } \\
\text { Eppendorf } 1101 \\
\text { LKB } 7400 \\
\text { SMA } 12 / 60\end{array}$ \\
\hline Protein, total & Biuret-reaction & $\begin{array}{l}\text { SMAC } \\
\text { SMA } 12 / 60 \\
\text { Greiner GSA II } \\
\text { ACA DuPont } \\
\text { LKB 2071/7.400 } \\
\text { Beckman DSA } \\
\text { C } 4 \text { Perkin Elmer } \\
\text { Vitatron UC } 200\end{array}$ \\
\hline Urea & $\begin{array}{l}\text { Diacetylmonoxime } \\
\text { Urease-diacetylmonoxime } \\
\text { Urease-Berthelot } \\
\text { Urease-Fawzett-Scott } \\
\text { Urease-glutamate dehydrogenase } \\
\text { Urease-nitroprusside-phenol }\end{array}$ & $\begin{array}{l}\text { SMA } 12 / 60 \\
\text { SMA } 12 / 60 \\
\text { Greiner GSA II } \\
\text { Eppendorf } 5020 \\
\text { LKB 2071/7400 } \\
\text { ACA DuPont } \\
\text { Autochemist }\end{array}$ \\
\hline Uric acid & $\begin{array}{l}\text { Phosphotungstic acid-hydrioxylamine } \\
\text { Uricăse } \\
\text { Uricasase-Ca }{ }^{++} \text {-neocuproin }\end{array}$ & $\begin{array}{l}\text { SMA } 12 / 60 \\
\text { Eppendorf } 1101 \\
\text { LKB } 2071 / 8600 \\
\text { Autochemist }\end{array}$ \\
\hline
\end{tabular}


Tab. 2. Variation coefficients (CV), level of significance between dose 0 and dose $1(0.71) \mathrm{mmol} / \mathrm{l})$ GOD $=$ glucose oxidase $F=\frac{s_{x}^{2}}{s_{y}^{2}}=$ critical values in 2 -factor variance analysis testing the null hypothesis at $0.05(=5 \%)$
significance levels.

\begin{tabular}{|c|c|c|c|c|c|c|c|c|c|c|c|}
\hline \multicolumn{3}{|c|}{ Glucose } & \multicolumn{3}{|l|}{ Uric acid } & \multicolumn{3}{|c|}{ Inorganic phosphorus } & \multicolumn{3}{|c|}{ Creatinine } \\
\hline $\begin{array}{l}\text { Lab. } \\
\text { No. }\end{array}$ & $\begin{array}{l}\mathrm{CV} \\
{[\%]}\end{array}$ & $\begin{array}{l}\text { level } \\
\text { of } \\
\text { significance }\end{array}$ & $\begin{array}{l}\text { lab. } \\
\text { No. }\end{array}$ & $\begin{array}{l}\mathrm{CV} \\
{[\%]}\end{array}$ & $\begin{array}{l}\text { level } \\
\text { of } \\
\text { significance }\end{array}$ & $\begin{array}{l}\text { lab. } \\
\text { No. }\end{array}$ & $\begin{array}{l}C V \\
{[\%]}\end{array}$ & $\begin{array}{l}\text { level } \\
\text { of } \\
\text { significance }\end{array}$ & $\begin{array}{l}\text { lab. } \\
\text { No. }\end{array}$ & $\begin{array}{l}\mathrm{CV} \\
{[\%]}\end{array}$ & $\begin{array}{l}\text { level } \\
\text { of } \\
\text { significance }\end{array}$ \\
\hline 1 & $\downarrow 10$ & 0.001 & 1 & $\downarrow 1.7$ & n.s & 1 & $\downarrow 1.5$ & n.s. & 1 & $\uparrow 4.9$ & 0.01 \\
\hline 3 & $\downarrow 19$ & 0.001 & 3 & $\downarrow 1.1$ & n.s. & 2 & $\downarrow 0.9$ & n.s. & 2 & $\uparrow 0.1$ & n.s. \\
\hline 6 & $\downarrow 1$ & n.s. & 7 & $\downarrow 1.6$ & n.s. & 5 & $\downarrow 1$ & n.s. & 3 & $\uparrow 9$ & 0.01 \\
\hline 7 & $\downarrow 15.4$ & 0.001 & 2 & $\uparrow 6.2$ & 0.001 & 7 & $\downarrow 2.4$ & n.s. & 4 & $\uparrow 2.2$ & 0.05 \\
\hline 8 & $\downarrow 7.8$ & 0.01 & 4 & $\uparrow \quad 5.3$ & 0.001 & 3 & & & 5 & $\uparrow 2.4$ & n.s. \\
\hline 2 & $\uparrow 4$ & 0.001 & 5 & $\uparrow \quad 5.4$ & 0.001 & 6 & 0 & & 6 & $\uparrow 2.3$ & n.s. \\
\hline 4 & $\uparrow \quad 2.1$ & 0.01 & 6 & $\uparrow 6.8$ & 0.001 & 8 & $\uparrow 4.8$ & 0.05 & 7 & $\uparrow 3.1$ & n.s. \\
\hline \multirow[t]{2}{*}{5} & $\uparrow 2$ & 0.001 & 8 & $\uparrow 55.3$ & 0.001 & & $\cdot$ & & 8 & $\uparrow 6.7$ & 0.01 \\
\hline & & $\mathrm{F}$ & & & $\mathbf{F}$. & & & $\mathbf{F}$ & & & $\mathbf{F}$ \\
\hline \multirow{2}{*}{\multicolumn{2}{|c|}{ All lab. }} & 4.44 & \multirow{2}{*}{\multicolumn{2}{|c|}{ all lab. }} & 11.4 & \multirow{2}{*}{\multicolumn{2}{|c|}{ all lab. }} & 0.013 & \multirow{2}{*}{\multicolumn{2}{|c|}{ all lab. }} & 1.80 \\
\hline & & 0.05 & & & 0.001 & & & n.s. & & & n.s. \\
\hline \multirow{2}{*}{\multicolumn{2}{|c|}{$\begin{array}{l}\text { Lab. } 1,3,7,8 \\
\text { (GOD Perid) }\end{array}$}} & 15.64 & \multirow{2}{*}{\multicolumn{2}{|c|}{ lab. $2-6$}} & 5.14 & \multirow{2}{*}{\multicolumn{2}{|c|}{ lab. $2,3,5,6$}} & 0.065 & \multirow{2}{*}{\multicolumn{2}{|c|}{ lab. $2-6$}} & 0.97 \\
\hline & & 0.001 & & & 0.01 & & & n.s. & & & n.s. \\
\hline \multirow{2}{*}{\multicolumn{2}{|c|}{$\begin{array}{l}\text { Lab. 2, 4-6 } \\
\text { (neocuproin) }\end{array}$}} & 0.41 & & & & & & & & & \\
\hline & & n.s. & & & & & & & & & \\
\hline
\end{tabular}

For other parameters, a more or less clear interference is noted.

\section{Glucose}

Glucose oxidase methods yield results that are low (22-25), neocuproin methods yield results that are high with both methods $(24,26,27)$; the variations are statistically significant.

\section{Uric acid}

Methods using uricase are not affected by ascorbic acid $(28,29)$ except when the second stage is based on copper reduction. Phosphotungstate techniques are affected significantly even at the lowest concentration of ascorbic acid $(5,23,25,30-32)$.

\section{Creatinine}

Three laboratories use methods without deproteinization. The interference is greater if measurement is preceded by dialysis. This might be due to a change in physical properties of the serum upon the addition of ascorbic acid (33-35).

\section{Inorganic Phosphate}

The dispersion of results is great, the observed variations do not appear to be significant.

\section{Second study}

The results of this second study are demonstrated by four graphs.

\section{Glucose (fig. 1)}

Neocuproine methods show increasing, glucose oxidasePerid methods decreasing values with increasing concentrations of ascorbic acid; the effect is already apparent at therapeutical levels of ascorbic acid.

Methods using glucose oxidase without indicator reaction, hexokinase/glucose-6-phosphate dehydrogenase or glucose dehydrogenase respectively, are not influenced by ascorbic acid.

\section{Uric acid (fig. 2)}

Phosphotungstate methods show increasing values with increasing concentrations of ascorbic acid.

Uricase methods do not behave in a uniform manner depending on the nature of the secondary reaction:

Uricase-hydrazone methods yield greatly decreased values, uricase neocuproin methods greatly increased values, being useless with higher concentrations of ascorbic acid.

The uricase/catalase/formaldehyde reaction (Kageyama) is not affected (36).

\section{Creatinine (fig. 3)}

Jaffe's reaction, used in mechanized analysis systems without deproteinization, is influenced by ascorbic acid in different ways. Methods with dialysis show a primary decrease followed by an elevation up to normal values. 


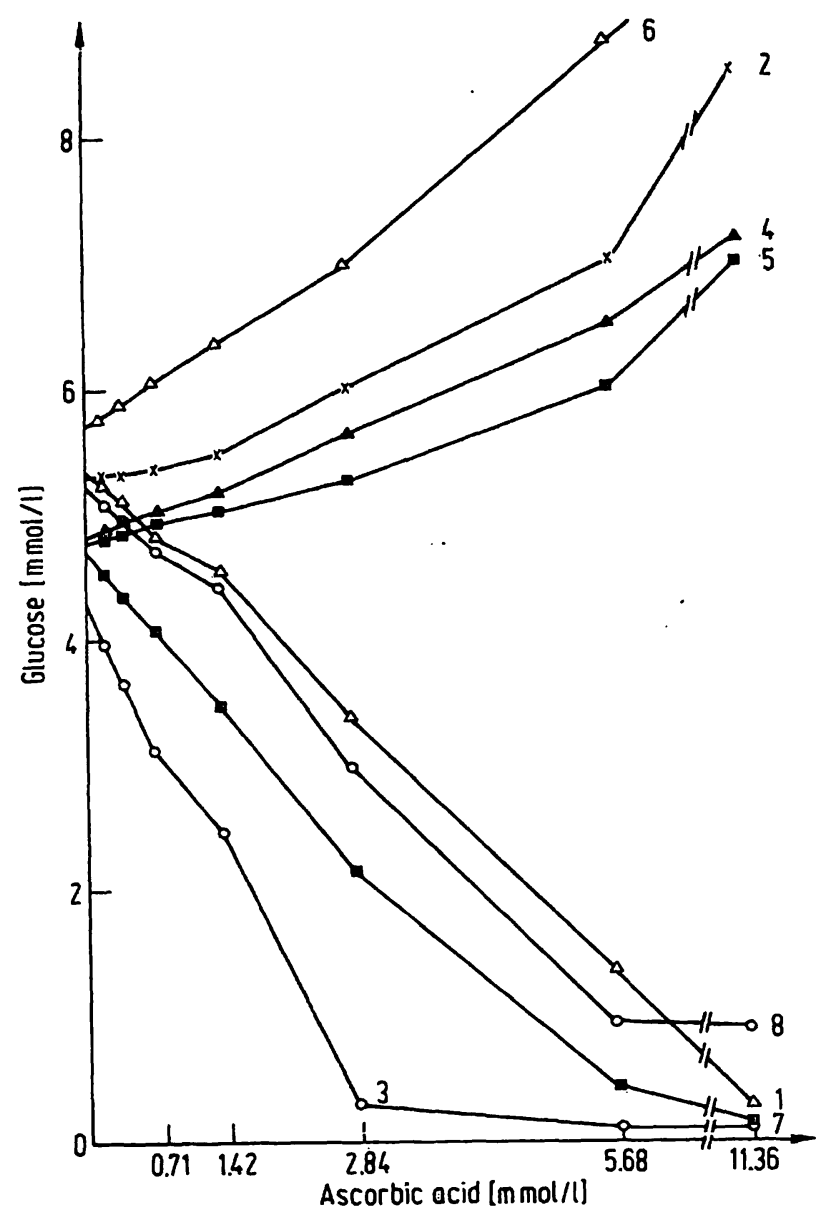

Fig. 1. Interference by ascorbic acid in glucose tests.

\begin{tabular}{lll}
$2,4,5,6$ & $\begin{array}{l}\text { Neocuproin } \\
\text { Glucose oxidase } \\
\text { Perid method } \\
\text { Boehringer ref. 15754 }\end{array}$ & $\begin{array}{l}\text { SMA 12/60 } \\
\text { Vitatron UC 200 S + } \\
\text { Digilog DRP 200 }\end{array}$ \\
3 & $\begin{array}{l}\text { Glucose oxidase-perid } \\
\text { Glucose oxidase- } \\
\text { peroxidase }\end{array}$ & $\begin{array}{l}\text { Technicon } \\
\text { LKB 7400 }\end{array}$ \\
7 & $\begin{array}{l}\text { Trinder method, } \\
\text { glucose oxidase }\end{array}$ & Gilford \\
\hline
\end{tabular}

Kinetic methods tend to elevated values with increasing amounts of ascorbic acid. All effects are more or less pronounced depending on the type of reagent or kit.

\section{Inorganic phosphate (fig. 4)}

$\mathrm{SnCl}_{2}$-hydrazine methods tend to lowered valuès with higher concentrations of ascorbic acid, but the difference is not significant.

Methods using hydroquinone-ascorbate, vanadate and p-methylaminophenol are not influenced by ascorbic acid.

\section{Discussion}

The spread of results obtained by the 8 laboratories was considerable. As in all surveys, there is primarily a problem of accuracy. Each laboratory was not expected to change its calibration. In a special trial, a serum

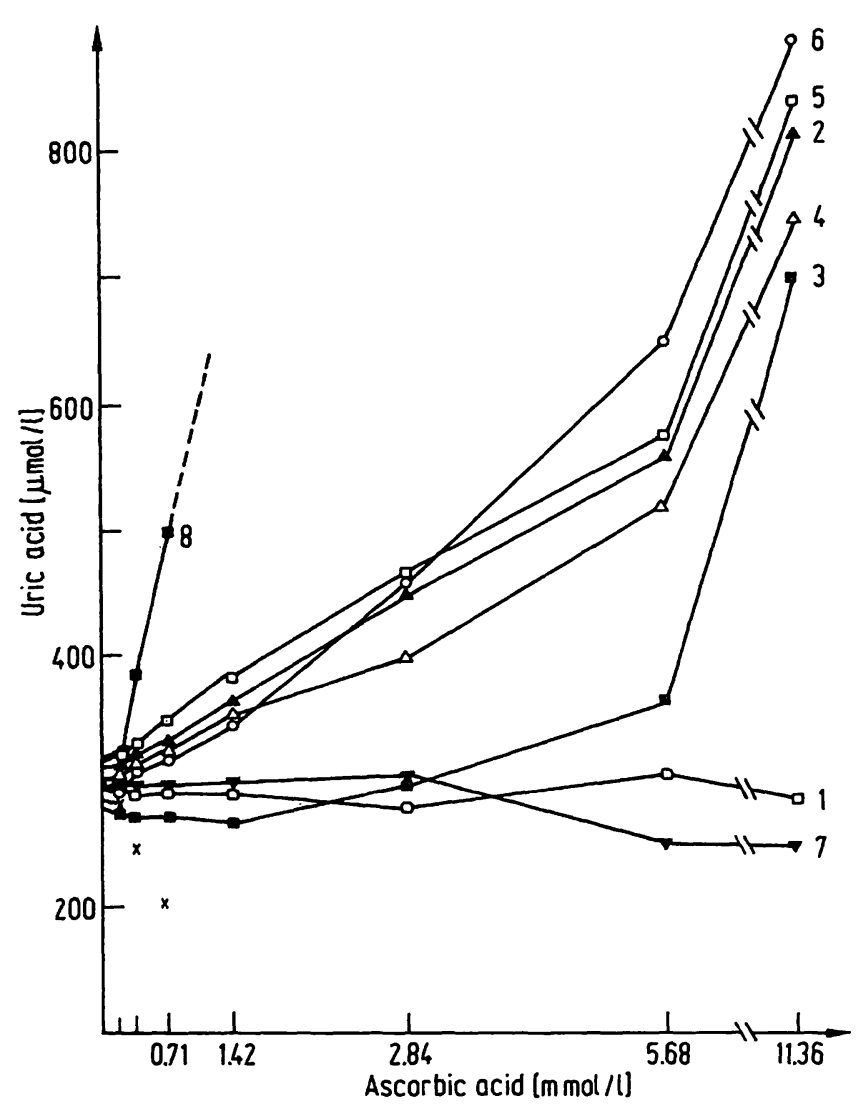

Fig. 2. Interference by ascorbic acid in uric acid tests.

\begin{tabular}{|c|c|c|}
\hline $2,3,4,5,6$ & $\begin{array}{l}\text { Phosphotungstic acid- } \\
\text { hydroxylamine }\end{array}$ & SMA $12 / 60$ \\
\hline 1 & $\begin{array}{l}\text { Enzymatic color test } \\
\text { Uricase, Boehringer } \\
\text { ref. } 15865\end{array}$ & Eppendorf 110 \\
\hline 7 & $\begin{array}{l}\text { UV test } \\
\text { Uricase-Boehringer } \\
\text { ref. } 15986\end{array}$ & $\begin{array}{l}\text { LKB 2071- } \\
\text { LKB } 8600\end{array}$ \\
\hline 8 & $\begin{array}{l}\text { Uricase- } \mathrm{Ca}^{++}-\text {neo- } \\
\text { cuproin }\end{array}$ & $\begin{array}{l}\text { Auto- } \\
\text { chemist }\end{array}$ \\
\hline 9 & Uricase-hydrazone & - \\
\hline
\end{tabular}

having an "assigned" value was integrated into the series and the results adjusted using this value. The addition of the drug in vitro did not appear to increase the dispersion. Therefore, depending on the analytical systems, principles of the methods, combinations of reaction steps, reagents, kits, and instruments, it is not possible to discuss in a simple way the factors concerned in the interference by ascorbic acid.

Methods that are not influenced by ascorbic acid are listed in table 3. These results agree with the existing literature. Table 4 shows those analytical methods for glucose, uric acid, and creatinine that are regarded as less recommendable. Concerning the risk of false interpretation of results, the concentrations and pharmacokinetic data for ascorbic acid in vivo cannot be neglected. The concentrations used in this study cover the zone of therapeutic values $(0-0.125 \mathrm{~g} / 1 \cong 0-0.71$ $\mathrm{mmol} / \mathrm{l})$ and likewise values which may be attained due to therapeutic accidents (21). To achieve more information it is necessary to perform studies in vitro as 


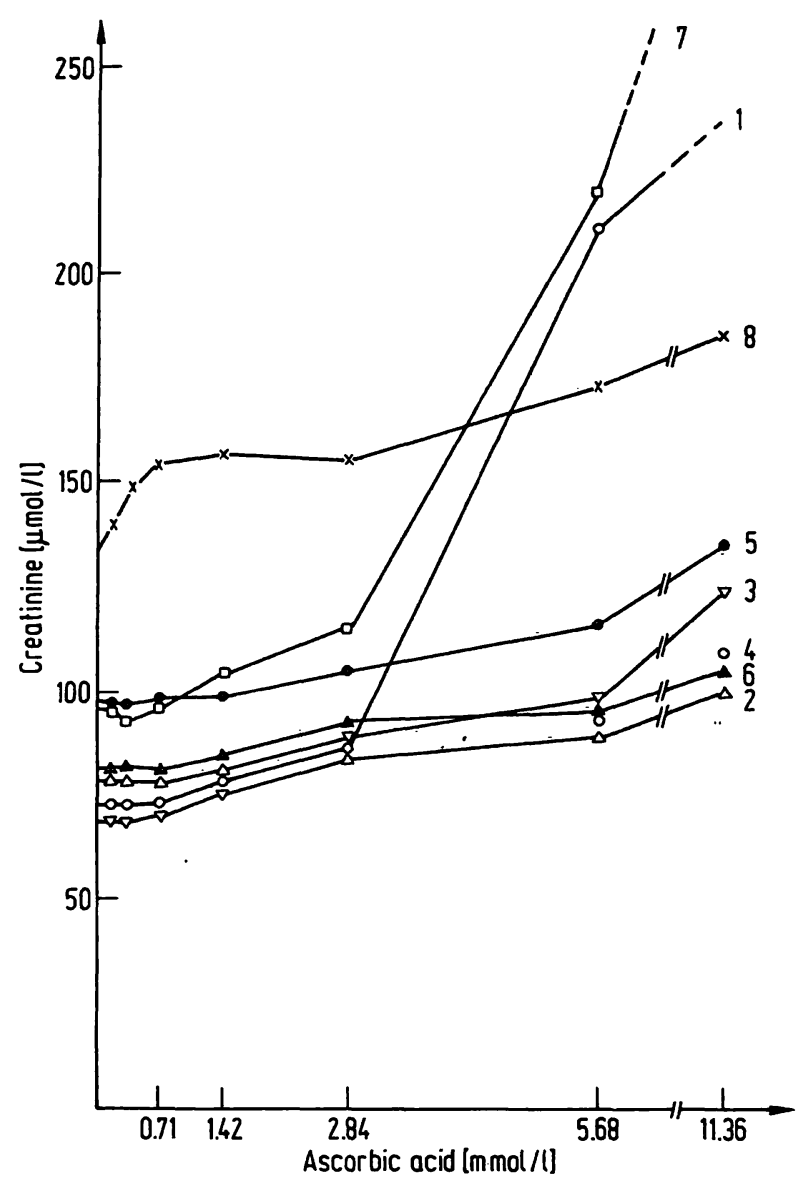

Fig. 3. Interference by ascorbic acid in creatinine tests.

$\begin{array}{lll}2,3,4,5,6 & \begin{array}{l}\text { Alkaline picrate + dia- } \\ \text { lysis (Jaffé) } \\ \text { Kinetic test-picric } \\ \text { acid Merckotest } \\ \text { ref. } 3385 \text { (without } \\ \text { deproteinisation) }\end{array} \\ \text { Kinetic test-picric acid } \\ \text { Merckotest ref. 3384 } \\ \text { (without } \\ \text { deproteinisation) } \\ \text { Alkaline picrate } \\ \text { (Jaffé) }\end{array}$

well as in vivo to link plasma concentrations of ascorbic acid to quantitative data of interferences in the analytical method. This aim overlaps with the field of "drug interactions" and is regarded as part of clinical pharmacology.

Restricting our attention to the basic program, we have been able to reduce errors and misleading conclusions to a minimum by exclusion of interlaboratory, interdisciplinary, economic and political-specific and unspecific interests and influences.

The coupling of a method to one instrument by a " manufacturer may force the responsible clinical chemist to use methods with a higher risk of drug interference: in such a case, method, kit or machine should be changed. Considering the consumption of ascorbic acid as vitamin tablets, juices, vegetables and fruits (including canned food with ascorbic acid as preservative), especially when there is a risk of infection the clinical chemist and
Tab. 3. Analytical methods not influenced by ascorbic acid.

\begin{tabular}{|c|c|}
\hline Objectives & Methods \\
\hline $\begin{array}{l}\text { Aspartate amino- } \\
\text { transferase }\end{array}$ & $\begin{array}{l}\text { UV-test, kinetic, opt. } 25^{\circ} \mathrm{C}^{1} \text { ) } \\
\text { UV-test, kinetic, non opt. } 37^{\circ} \mathrm{C}^{1} \text { ) } \\
\text { UV-test, end point }{ }^{1} \text { ) } \\
\text { Colorimetric test }{ }^{1} \text { ) }\end{array}$ \\
\hline Phosphatase, alkaline & $\begin{array}{l}\left.\text { Kinetic-test, } p \text {-nitrophenyl phosphate }{ }^{1}\right)^{2} \text { ) } \\
\text { Kinetic-test, phenyl disodium } \\
\text { phosphate }{ }^{2} \text { ) } \\
\text { Kinetic-test, 4-aminoantipyrin-disodium- } \\
\text { hydrogencarbonat }{ }^{2} \text { ) }\end{array}$ \\
\hline Bilirubin & $\begin{array}{l}\text { Sulfanilic acid-caffein }{ }^{1} \text { ) } \\
\text { Dichloraniline-nitrite }{ }^{1} \text { ) }\end{array}$ \\
\hline Calcium & $\begin{array}{l}\text { o-Cresolphthalein-complexon } \\
\text { Thymolphthalein-complexon } \\
\text { Flamephotometry } \\
\text { Calcein-titration }\end{array}$ \\
\hline Cholesterol, total & $\begin{array}{l}\text { Acetic anhydride-acetic acid } \\
\text { Huang, Liebermann-Burchard, Watson } \\
\text { Esterase/oxidase-catalase } \\
\text { Esterase-hydrogen peroxidase }\end{array}$ \\
\hline Creatinine & $\begin{array}{l}\text { (Alkaline picrate (Jaffé) without } \\
\text { deproteinisation or with dialysis) }\end{array}$ \\
\hline Glucose & $\begin{array}{l}\text { Hexokinase/glucose-6-phosphate } \\
\text { dehydrogenase } \\
\text { Glucose dehydrogenase }\end{array}$ \\
\hline Phosphate, inorganic & $\begin{array}{l}\text { Molybdate-hydroquinone-ascorbate } \\
\text { Molybdate-p-methylaminophenolsulfate } \\
\text { Molybdate-vanadate }\end{array}$ \\
\hline Protein, total & Biuret-reaction \\
\hline Urea & $\begin{array}{l}\text { Diacetyl-monoxime methods } \\
\text { Urease-Berthelot } \\
\text { Urease-glutamate dehydrogenase } \\
\text { Urease-nitroprusside-phenol }\end{array}$ \\
\hline Uric acid & Unicase, Kageyama's reaction \\
\hline
\end{tabular}

() = Interference at higher concentrations of ascorbic acid.

1) No clear conclusion by reason of non systematic variations.

$\left.{ }^{2}\right)$ No clear conclusion by reason of technical problems.

Tab. 4. Analytical methods regarded as being less recommendable.

\begin{tabular}{|c|c|}
\hline Objective & Method \\
\hline Glucose & $\begin{array}{l}\text { Glucose oxidase methōds with indicător } \\
\text { reaction } \\
\text { Neocuproin methods }\end{array}$ \\
\hline Uric acid & $\begin{array}{l}\text { All phosphotungstic methods } \\
\text { Uricase methods with copper reduction } \\
\text { Uricase-hydrazone methods }\end{array}$ \\
\hline Creatinine & $\begin{array}{l}\text { Picrate methods without deproteini- } \\
\text { sation preceded by dialysis } \\
\text { (Picric acid, kinetic tests, depending on } \\
\text { different type of reagents or kits) }\end{array}$ \\
\hline
\end{tabular}

( ) = Interference at higher concentrations of ascorbic acid. 


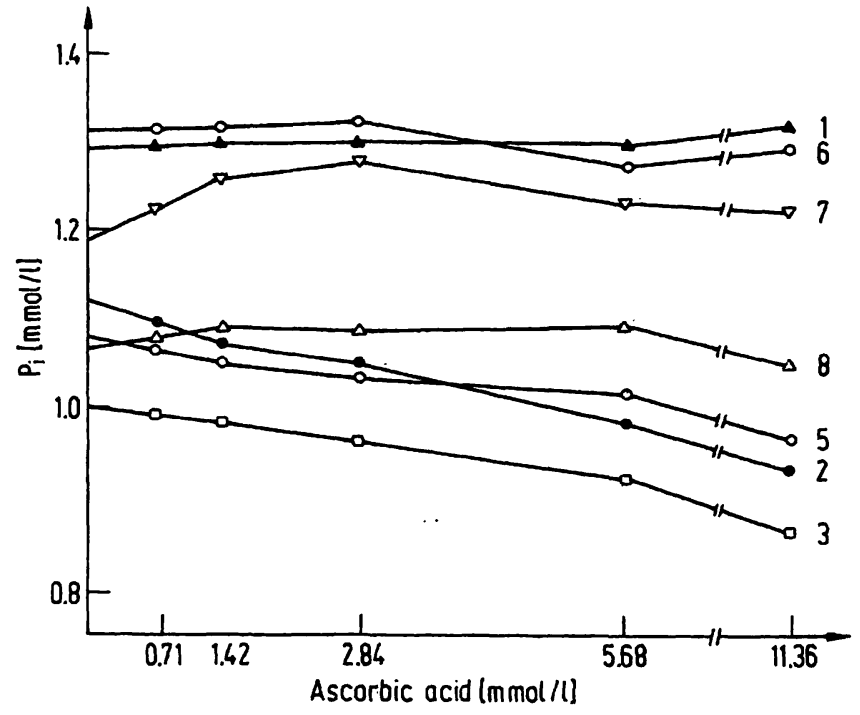

Fig. 4. Interference by ascorbic acid in phosphate tests.
$2,3,5,6$
Molybdic acid-
stannous chloride-
SMA $12 / 60$
hydrazine
1 Molybdate-p-methyl- Eppendorf
aminophenol Sulfate, 1101
Merckotest ref. 3331
7 Molybdate-vanadate,
Boehringer ref. 15920
8 Molybdate-hydro-
quinone

\section{LKB 7400}
Autochemist

physician may indeed expect ascorbic acid interference in some of his analyses. Part of the aim of this EuropeanCommunities-study was to assist his decision in using more reliable methods.

The study will be concluded by pilot investigations on the influence of ascorbic acid on the qualitative and semiquantitative determination of urinary glucose, by glucose oxidase-paper strip combinations.

This study has been partially supported by the European Communities.

\section{Dr. K. L. Lauert}

Commission des Communautés Europeénnes

Bureau Communautaire de Référence

Rue de la Loi 200

Joyeuse Entree 4-21

B-1040 Bruxelles

Prof. G. Siest

Laboratoire de Biochemie - Faculté des Sciences

Pharmaceutiques et Biologiques

7 , rue Albert Lebrun

B.P. 3102

F-54013 Nancy cedex

Dr. Walter Appel (for the authors)

Priv. Doz., Dipl. Chem

Zentrallaboratorium der St.-Vincentius-Krankenhäuser

D-7500 Karlsruhe

\section{Annexe}

General protocol of analytical interference by ascorbic acid

6 specimens were analysed in duplicate on each of three days.

\section{Materials provided}

- Ascorbic acid

- Bottles lyophilised serum (to make $10 \mathrm{ml}$ after reconstitution)

\section{Procedure}

1. Prepare a $11.36 \mathrm{mmol} / 1(2 \mathrm{~g} / \mathrm{l})$ ascorbic acid solution in redistilled water

2. Preparation of overloaded serum

a) Using this solution daily reconstitute two bottles of lyophilised serum (using $10 \mathrm{ml}$ )

b) Mix the contents of the two bottles

3. Preparation of control serum

a) Dissolve four bottles of lyophilised serum daily in redistilled water (using $10 \mathrm{ml}$ )

b) Mix the contents of the four bottles

4. Preparation of specimens for analyses

Prepare the following dilutions:

\begin{tabular}{lllll}
$\begin{array}{l}\text { Specimen } \\
\text { No. }\end{array}$ & $\begin{array}{l}\text { Concentrations of } \\
\text { ascorbic acid obtained }\end{array}$ & $\begin{array}{l}\text { Overloaded } \\
\text { serum }\end{array}$ & $\begin{array}{l}\text { Control } \\
\text { serum }\end{array}$ \\
& mmol/1 & $\mathrm{g} / \mathrm{l}$ & & \\
\hline 1 & 0 & 0 & $0 \mathrm{ml}$ & $8 \mathrm{ml}$ \\
2 & 0.14 & 0.025 & $0.1 \mathrm{ml}$ & $7.9 \mathrm{ml}$ \\
3 & 0.43 & 0.075 & $0.3 \mathrm{ml}$ & $7.7 \mathrm{ml}$ \\
4 & 0.71 & 0.125 & $0.5 \mathrm{ml}$ & $7.5 \mathrm{ml}$ \\
5 & 1.42 & 0.25 & $1 \mathrm{ml}$ & $7 \mathrm{ml}$ \\
6 & 2.84 & 0.5 & $2 \mathrm{ml}$ & $6 \mathrm{ml}$ \\
\hline
\end{tabular}

N.B.: Prepare all serum and the ascorbic acid solution fresh daily

\section{Assesment of interferences}

On each of the above dilutions perform the analyses, in duplicate and in order of increasing concentrations

6. Results

a) Specify the methods and instruments used

b) Indicate the analytic variation coefficient of your technique in the zone of values we are concerned with. 


\section{References}

1. Appel, W., Wirmer, V. \& Ebenezer, S. (1968), Anaesthesist 3, 95-105.

2. Appel, W. (1973), Beeinflussung klinisch-chemischer Meßergebnisse durch Arzneimittel, in Optimierung der Diagnostik (Lang, H., Rick, W. \& Roka, L., eds.) p. 135, Merck-Symposion, Springer-Verlag, Berlin.

3. Delwaide, P. A., Jadin, A. \& Heusghem, C. (1973), Interferences des médicaments sur les déterminations effectuées en chimie clinique, in Les effets indésirables des médicaments (Heusghem, C. \& Lechat, P., eds.), pp. 698-710, Masson et Cie., Paris.

4. Elking, M. P. \& Kabat, H. F. (1969), Am. J. Hosp. Pharmacy $25,485-519$.

5. Hansten, P. D. (1973), Drug interactions, Lea and Febiger, Philadelphia.

6. Lingaerde, P., Adlercreutz, H., Hjelm, M., Holmgard, A. \& De Verdier, C. H. (1973), in Progress in quality control in clinical chemistry pp. 211-217 (Anido, G., Van Kampen, E. J. \& Rosalki, S. B., eds.), Hans Huber Publ., Bern.

7. Loppinet, V., Siest, G. \& Lahrichi, M. (1976), Influence of electronic, steric and conformational factors of the membrane interference of drugs: Application of the effect of the phenothiazine structure on granulocytes, in Drug interference and drug measurement in clinical chemistry, (Siest, G. \& Young, D. S., eds.) pp. 58-66, Karger Publ., Bâle.

8. Lubran, M. (1969), Med. Clin. N. Amer. 53, 211-222.

9. Schneiderman, L. S., Desalvo, L., Baylor, S. \& Wolf, P. (1972), Arch. Intern. Med. 129, 88-96.

10. Schwartz, M. K. (1973), Interferences in diagnostic procedures, in Advances in clinical chemistry (Bodansky, O. \& Latner, A. L., eds.) Vol. 16, pp. 1-33, Academic Press, New-York-London.

11. Siegenthaler, N., Beckerhoff, R., Wursten, D. \& Zimmermann, K. (1973), Medikamentöse Nebenwirkungen durch Interferenz mit geregelten biologischen Systemen, in Optimierung der Diagnostik (Lang, H., Rick, W. \& Roka, L., eds.) p. 121, Merck-Symposion, Springer-Verlag, Berlin.

12. Siest, G. (1973), Interferences of drugs in the biological processes, in Reference values in human chemistry, p. 283 , Karger Publ., Bâle.

13. Siest, G., Batt, A. M., Galteau, M. M., Weber, M. \& Tridon, P. (1974), Therapie 29, 907-914.

14. Siest, G. \& Young, D. S. (1976), Drug interferences and drug measurement in clinical chemistry, Karger Publ., Bâle.

15. Sunderman, F. W. (1970), Crit. Rev. Clin. Lab. Sci. l, 427-449.

16. Van Peenen, M. J. \& Files, J. B. (1969), Am. J. Clin. Pathol. $52,666-670$.
17. Wepler, R. \& Rommel, K. (1973), Dtsch. Med. Wochenschr. 98,2307-2311.

18. Young, D. S. (1972), Modification of serum-enzyme activity by therapeutic drugs, pp. 282-289, Karger Publ., Bâle.

19. Young, D. S., Pestaner, L. C. \& Gibberman, V. (1975), Clin. Chem. 21, 1D-432D.

20. Bureau Communautaire de Référence, sous-groupe de travail "Drug Interference in Clinical Chemistry" Appel, W. (Karlsruhe), Blijenberg, B. G. (Rotterdam), Capolaghi, B. (Nancy), Galteau, M. M. (Nancy), Heusghem, C. (Liège), Hjelm, M. (Odense), Lauer, K. L. (Bruxelles), Le Perroñ; B. (Nancy), Love, W. C. (Dublin), Royer, R. J. (Nancy), Siest, G., Président, (Nancy), Tognoni, G. (Milañ) \& Wilding, P. (Birmingham) (1976), in Drug interferences and drug measurement in clinical chemistry (Siest, G. \& Young, D. S., eds.) pp. 1-9, Karger Publ., Bâle.

21. Appel, W. (1976), Medizin 4, 1824-1832.

22. Romano, Anna, T. (1973), Clin. Chem. 19, 1152-1157.

23. Constantino, Norma, V. \& Kabat, H. F. (1973), Am. J. Hosp. Pharmacy 30, 24-71.

24. Pennock, C. A., Murphy, D., Sellers; J. \& Long Don, K. J. (1973), Clin. Chim. Acta 48, 193-201.

25. Young, D. S. \& Panek, E. (1976), Effects of drugs on the analytical procedures of a multitest analyzer, in Drug interferences and drug meàsurement in clinical chemistry (Siest, G. \& Young, D. S., eds.) pp. 10-20, Karger Publ., Bâle.

26. Bailly, M. (1972), Perturbations des résultats d'analyses biochimiques provoquées par la thérapeutique, Compterendus des Journées Pharmaceutiques Internationales de Paris, p. 127.

27. Barry, S. \& Reiss, Ph. D. (1975), J. Clin. Pharmacol. 15, 135-138.

28. Kabasakalian, P., Kalliney, S. \& Westçott, A. (1973), Clin. Chem. 19, 522-524.

29. Morin, L. G. (1974), Clin. Chem. 20, 51-56.

30. Kelley, W. N. (1975), Ann. Rev. Pharmacol. 15, 327-350.

31. Lum, G. \& Gambino, R. S. (1973), Clin. Chem. 19, $1184-1186$.

32. Singh, H. P., Hebert, M. A. \& Galteau, M. M. (1972), Clin. Chem. 18, 137-148.

33. Haugh, H., Dorlöchter, E. \& Hermann, G. (1972), Diagnostik $5,85-88$.

34. Heinegard, D. \& Tiderstrom, G. (1973), Clin. Chim. Acta.43, 305-310.

35. Mitchell, R. J. (1973), Clin. Chem. 19, 408-410.

36. Mathies, H., Staehler, F., Wittner, H. \& Vollmar, J. (1974), Med. Klinik 69, 607-612.

Priv.-Doz. Dr. W. Appel

Zentrallaboratorium

St. Vincentius-Krankenhäuser

Südendstr. 32

D-7500 Karlsruhe 